1 ENSURING HIGH QUALITY CLINICAL AND PATIENT REPORTED OUTCOMES - A RAG RATING FOR SURGEONS CLINICAL GOVERNANCE

Alexander Silvester, John Woodhouse. SpMedica, UK, Clarity and Partnership, UK

10.1136/leader-2020-FMLM.1

Clinical governance and maintaining high quality clinical and patient reported outcomes (PROMS) are crucial within the healthcare sector. The Francis report (2015) and the Paterson inquiry (2020) highlighted systemic patient safety risks within the NHS and private healthcare sector. As a large independent organisation, providing NHS cataract surgery from 17 hospitals performed by over 30 Consultant Ophthalmologists, a system was required to benchmark surgeons. Previous measures used in surgeon appraisal and revalidation provide only a snapshot of selected PROMs and outcomes. Methodology A red, amber, green (RAG) rating was developed using clinical and PROMs following cataract surgery. Data was collated from the electronic medical records following review by an independent optometrist. An algorithm was constructed based on national standards presented as a quarterly RAG score. Surgeons scoring red received an intervention from the Medical Director. Results RAG rating has ensured the maintenance of high clinical and PROMs. Often working independently, the RAG rating has enabled cataract surgeons to benchmark against peers. We have examples of surgeons improving their clinical outcomes and patient interactions after successive RAG ratings that have benchmarked them against their peers and nudged them to change their behaviour. Discussion Through regular feedback and benchmarking surgeons against their peers, this system has improved the quality of surgical outcomes and patient interactions. RAG rating combining clinical skills and softer communication skills and behaviour can be used to continuously monitor and benchmark surgeons. Such RAG ratings can highlight to managers surgeons who require an intervention, and to monitor change following the intervention. This is particularly useful for a multi-site organisation that wants to ensure high quality surgical outcomes consistently throughout their organisation.

\section{LEADERSHIP IN CLINICAL DECISION SUPPORT: IMPROVING ACCESS AT THE POINT OF CARE}

Elin Goodwin, Kieran Walsh. BMJ Knowledge Centre, UK

\subsection{6/leader-2020-FMLM.2}

BMJ Best Practice provides clinical decision support that is evidence based, continually updated and practical. Clinical decision support must be provided at the point-of-care if it is to have maximum impact. Our users gave us feedback that internet access or Wi-Fi was not always reliable at their workplace and so an offline app was required. In light of this feedback, we built a new BMJ Best Practice app that was based explicitly on user needs. This included a resource that could be downloaded quickly; that would use minimal space; that would contain all the features of the live website; and that would enable users to find answers to their questions within seconds. Through a process of continual improvement we launched a new app that would better satisfy user needs. We monitored user feedback to the new app. We made incremental improvements to the app in light of this feedback. We measured the effect of the improvement by monitoring feedback to the app. The app is now receiving a rating of 4.7 out of 5 . We have also analysed qualitative feedback. This is now overwhelmingly positive. Qualitative feedback shows that users are now able to use BMJ Best Practice at the point-ofcare. Users are also finding the tool to be a quick method of getting an answer. We have learned the importance of the leadership team basing all improvements on the stated content or technology needs of users. We have also learned that this is an ongoing process. When setting out on this work, we had a clear aim to achieve digital leadership in online clinical decision support. We have found that this can only be achieved by basing all plans on the needs of doctors on the frontline who are responsible for delivering care to patients.

\section{Leading innovation and improvement}

\section{MAKING HEALTHCARE INFORMATION AVAILABLE AND ACCESSIBLE TO ETHNIC MINORITY PEOPLE ( NEPALESE PEOPLE ) LIVING IN HONG KONG}

Lachhemi Rana. Hong Kong

\subsection{6/leader-2020-FMLM.3}

Nepalese people consist of part of the $8 \%$ which is the total percentage of ethnic minority living in Hong Kong. Many Nepalese do not understand English and most of the elderlies are illiterate, depriving them the access to basic health information and also the health system provided by the Hong Kong government. Many Nepalese construction workers are unable to make informed career choices and are often inflicted with long term occupational health disease, while others are missing out many government health schemes like; health care vouchers for the elderly, free influenza and pneumococcal vaccines for children and elderly, free occupational health check up for the construction worker etc. These problems were assessed when I had personally worked and participated in different Non-governmental organisations's projects, workshops, programs and by even working in private clinic in Hong Kong for more than half a decade. For the solution, I had applied for the 'Community Involvement Broadcasting service' (CIBS) project that is run by Radio Television Hong Kong under Hong Kong Government. I produced many health related radio programs in Nepalese language after going through nationwide competition and also with votes from community people. This allowed people to have access to the health information in Nepali language as all the programs are recorded in the podcast format and are accessible to their dispense. A surge in number of queries about health related information was noted in many local NGOs. After completion of each program, a survey was carried out among the crew members and was submitted to RTHK. A separate survey was done with the listeners and guests. The problem still remained with the usage of the radio app on the mobile devices of the elderly group who were always in need of assistance. We can achieve better health for the community by taking the leadership role, looking for available solution and involving people of the community.

Community leadership 